\title{
Numerical Simulation of Three Phase Displacement Characteristics of Foam Fluid In Homogeneous Porous Media
}

\author{
Du D.X, Zhang D \\ 1. College of Electromechanical Engineering, Qingdao University of Science and \\ Technology, Qingdao 266061, China \\ 2. Geo-Energy Research Institute, Qingdao University of Science and Technology, Qingdao \\ 266109, China \\ E-mail: du-dongxing@163.com , Tel: 0532-88956166
}

\begin{abstract}
Foam technology has found wide applications in enhanced oil recovery and greenhouse geological storage. In this paper, a numerical simulate is carried out with the stochastic bubble population balance model on the foam three phase displacement process in a homogeneous oil/water/gas coexistence porous media of liquid. The effects of the maximum equilibrium bubble density $n_{\max }$ and the foam generation rate $\mathrm{Kg}$ on foam displacement process is mainly discussed. Numerical results indicate that the oil phase can be displaced well by foam fluid. Larger $n_{\max }$ values lead to higher apparent viscosity of foam and higher the pressure difference, and with the increase of $\mathrm{Kg}$, the number of foam can reach a balance in a short distance, so it has better displacement effect on the oil phase components. The results obtained in this paper have a certain guiding role in understanding the enhanced oil recovery mechanism of foam fluid.
\end{abstract}

Keywords: porous media; foam fluid; three-phase displacement; numerical simulation

\section{Introduction}

In the process of oil exploitation, foam technology is widely used to enhance the industrial practice of oil recovery (EOR). The gas flooding usually leads to lower oil displacement efficiency due to the gravity segregation and the channeling of gas in high permeability strata, while foam technology can significantly increase the viscosity of the gas phase, control the displacement gas well and final improve the oil recovery ${ }^{[1-6]}$.

In order to describe the seepage characteristics of foam liquid in homogeneous porous media successfully, we must conduct in-depth research on it. The displacement characteristics of foam in porous media can be analyzed, simulated and predicted simply and reliably by numerical methods. Some researchers use the assumption of single phase non Newtonian fluid to simulate the foam rheological properties ${ }^{[7-9]}$, but the method is lack of generality and can not reflect well the percolation mechanism of foam in porous media and predict correctly displacement characteristics. In the rheological model based on two-phase fluid assumption, the number of bubbles $n_{f}$ is a very important parameter. Among the current foam number models, the most famous are the phase-separation flow model and the bubble number conservation model. The phase separation flow model is proposed by Rossen ${ }^{[10-14]}$, and the conservation model of the number of bubbles are proposed by Patzek and Kovscek etc. ${ }^{[15-18]}$.Although based on the dynamic balance of bubble density, the conservation model is too difficult to apply in practice due to the excessive number of parameters in the model. Therefore, the more successful commercial software for multiphase flow modeling of reservoirs, such as Eclipse ${ }^{[19]}$ and CMG's STARS ${ }^{[20]}$, does not calculate the seepage flow rate by calculating the apparent viscosity of foam, but directly revises the flow of the foam phase.

Recently, Zitha and Du (2010) ${ }^{[21-22]}$ developed a new conservative model for the number of random foams that moves in foams in porous media. The stochastic population balance model is based on the following basic assumptions:

(1)As a complex two phase fluid system, the rheological properties of foam are described by HerschelBulkley model.

(2)The foam rheology essentially depend on the foam density (number of bubbles per unit volume of porous media).

(3)The generation of foam could be treated as a stochastic process and the kinetics of foam generation obeys a simple exponential growth law. 
Compared with the traditional quantitative equilibrium model, the stochastic population balance model consists of two basic parameters (foam generation rate of $\mathrm{Kg}$ and maximum bubble number of $n_{\infty}$ ), which are more easily determined through experiments.

In this paper, the numerical simulation of the displacement process of gas, water and oil three-phase in homogeneous porous media is carried out by using the black oil model ${ }^{[23-26]}$ and the stochastic population balance model. In the numerical simulation process, the three phase displacement process of foam liquid in oilwater saturated porous media is mainly analyzed. At the same time, the numerical simulation results of foam displacement and gas displacement are compared.

\section{Numerical simulation}

\subsection{Computational domain}

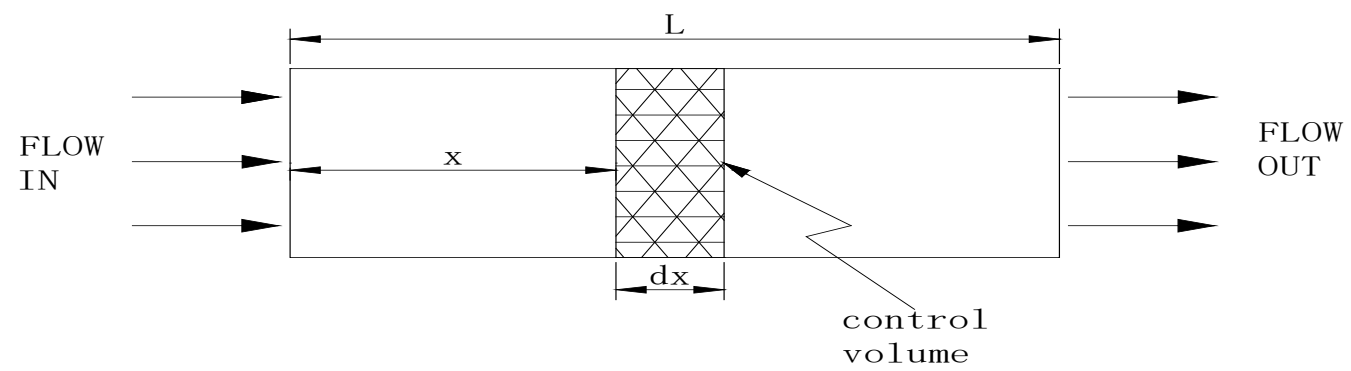

Fig.1. Computational domain

Fig.1. shows a homogeneous porous medium with a length of $L$, a porosity of $\Phi$ and a permeability of $K$ in the calculation area of this paper. This simulation is the foam displacement process of three phases of gas, water and oil along the sand core in one dimensional case, with gas injection from the left side at a certain speed $u$ to the right side. The length of the porous medium is $L=0.2 \mathrm{~m}$. The initial condition of the calculation is $40 \%$ water, $50 \%$ oil, $10 \%$ gas in the porous medium with a system back pressure of $0.1 \mathrm{MPa}$ (atmospheric condition). Gas injection rate is $0.005 \mathrm{~m} / \mathrm{s}$ and the velocities of the aqueous and oil phases are both set to $0 \mathrm{~m} / \mathrm{s}$. In the outlet, the pressure of the oil phase is set to be constant of $0.1 \mathrm{MPa}$ in the numerical simulation.

\subsection{The governing equations with stochastic population balance model}

Ignoring the gravity, the partial differential equation of the foam number conservation model ${ }^{[23]}$ is:

$$
\left\{\begin{array}{c}
\phi \frac{\partial\left(\rho_{i} S_{i}\right)}{\partial t}+\nabla \cdot\left(\rho_{i} \mathbf{u}_{i}\right)=0 \\
\mathbf{u}_{i}=-\lambda_{i} \nabla P_{i} \\
\phi \frac{\partial\left(n S_{f}\right)}{\partial t}+\nabla \cdot\left(n \mathbf{u}_{f}\right)=\phi S_{f} K_{g}\left(n_{\infty}-n\right)
\end{array}\right.
$$

Auxiliary equations are:

$$
S_{w}+S_{g}+S_{o}=1 ; \quad p_{\text {cow }}=p_{o}-p_{w}=f\left(S_{w}, S_{g}\right) ; \quad p_{c o g}=p_{g}-p_{o}=f\left(S_{w}, S_{g}\right)
$$

Where $\rho_{i}, S_{i}, u_{i}, \lambda_{\mathrm{i}}, P_{i}$ respectively refers to the $i$-phase fluid density, saturation, velocity, fluidity and total pressure, where $i \in\{w, f, o\}$ (subscript $f$ refers to the bubble). $K_{g}$ and $n_{\infty}$ are foam-related parameters that can be easily measured experimentally. In this simulation analysis, the aqueous phase is assumed to be incompressible and the foam conforms to the ideal gas law. $n_{\infty}$ is approximately equal to the pore volume of foam and porous media at steady state, which is: $n_{\infty} \approx S_{f} \varphi / r^{3}$.

In the auxiliary equation, $p_{c o w}, p_{c o g}$ refers to the capillary pressure between oil-water phases and oil-gas phases. Among them, the capillary pressure of oil-water and oil-gas are the function of liquid-phase saturation $S_{w}$ and gas-phase saturation $S_{g}$, respectively. The capillary pressure is taken as 0 in this simulation for simplification, due to the capillary pressure is relatively small.

The mobility $\lambda_{i}$ of the $i$-phase fluid is defined as:

$$
\lambda_{i}=\frac{k_{i}}{\mu_{i}}=\frac{k k_{r i}}{\mu_{i}}
$$

Where $k_{r i}$ is relative permeability of the $i$-phase fluid, $\mu_{i}$ - the viscosity of the fluid, and 


$$
\mu_{f}=\mu_{g}+\alpha \frac{n}{u_{f}^{c}}
$$

Where $\alpha$ and $c$ are constants and $\mathrm{n}$ is the bubble density.

The relative permeability $k_{r i}$ fluid expression is ${ }^{[9]}$ :

$$
k_{r o}=\left(\frac{S_{o}}{1-S_{w c}}\right)^{\lambda} ; k_{r g}=\left(\frac{S_{g}}{1-S_{w c}}\right)^{\lambda} ; \quad k_{r w}=\left(\frac{S_{w}-S_{w c}}{1-S_{w c}}\right)^{\lambda}
$$

Where $S_{w c}$ refers to the residual water saturation, take 0.23 ; $\lambda$ is a constant of 3.0 .

The employed parameters are listed in Table 1.

Table 1 fluid and rock properties parameters

\begin{tabular}{cccc}
\hline Parameter & Value & Parameter & Value \\
\hline$S_{w c}$ & 0.23 & $\mu_{g}(\mathrm{~Pa} \cdot \mathrm{s})$ & $18.1 \times 10^{-6}$ \\
$\phi$ & 0.23 & $\mathrm{a}$ & $8.6 \times 10^{-7}$ \\
$K\left(\mathrm{~m}^{2}\right)$ & $2.0 \times 10^{-12}$ & $\lambda$ & 3.0 \\
$\mu_{w}(\mathrm{~Pa} \cdot \mathrm{s})$ & $1004.0 \times 10^{-6}$ & $\mathrm{c}$ & 0.333333 \\
\hline
\end{tabular}

Note: The parameters identified in Table 1 satisfy Bentheimer sandstone ${ }^{[27]}$.

\subsection{Method of numerical solution}

In the numerical solution, we assume that: no gas dissolved in oil phase with $R_{s}=0$, the water and oil phase are incompressible with $B_{o}=1, B_{w}=1$.

We solve equation $(1) \sim(2)$ using IMPES method, which divides the numerical procedure into three steps (superscript $n$ on behalf of time):

(1)The oil phase pressure $P_{o}^{n}$ at time $n$ is implicitly calculated from the oil phase pressure $P_{o}^{n-1}$ at the previous time, and the gas phase pressure $P_{f}^{n}$ at the time $n$ which can be obtained from the sum of $P_{o}^{n}$ and $P_{c o g}^{n}$, and the the water phase pressure $P_{w}^{n}$ at time $n$ can be found from the difference between $P_{o}^{n}$ and $P_{\text {cow }}^{n}$, where $P_{\text {cog }}^{n}, P_{\text {cow }}^{n}$ is known.

(2) The water phase saturation distribution $S_{w}^{n+1}$ at time $n+1$ can be explicitly obtained from $S_{w}^{n}$ and $P_{w}^{n}$.

(3) The oil phase saturation distribution $S_{o}^{n+1}$ at time $n+1$ can be explicitly determined by $S_{o}^{n}$ and $P_{o}^{n}$.

(4) The foam density distribution $n^{n+1}$ at time $n+1$ can also be determined explicitly using known values $n^{n}, P_{f}^{n}, S_{f}^{n}, S_{f}^{n+1}$

\section{Results and analysis}

\subsection{Foam three phase displacement process in homogeneous porous medium $\left(K g=0.5, n_{\max }=200\right)$}

Fig.2. shows the typical curves of water phase pressure, oil/water/gas phase saturation and foam density parameters along the sample in the transient displacement times of $10 \mathrm{~s}, 20 \mathrm{~s}$, 40s and 50 s respectively. Numerical calculations are carried out under the condition of $K g=0.5$ and $n_{\max }=200$, and the typical foam three phase displacement behavior can be observed from the figure:

(1) The water phase pressure drop increases gradually along with the displacement process. At $t=40 \mathrm{~s}$ and $50 \mathrm{~s}$, the pressure difference is basically kept around $0.37 \mathrm{MPa}$, indicating that the foam has completed the breakthroughs and achieved fully development at this time.

(2) At the designated relative permeability functions, the foam fluid mainly displaces the oil phase rather than water phase during the displacement process. After foam breakthrough, the foam (gas) saturation in the medium increases to $0.5-0.65$, the oil saturation decreases to $0.1-0.2$, which the water saturation is maintained around 0.4 . The calculation results show that the injection of foam can efficiently displace the oil phase.

(3) Along with the displacement time, the foam density increases along with the change of oil, water and gas three phase saturation. 


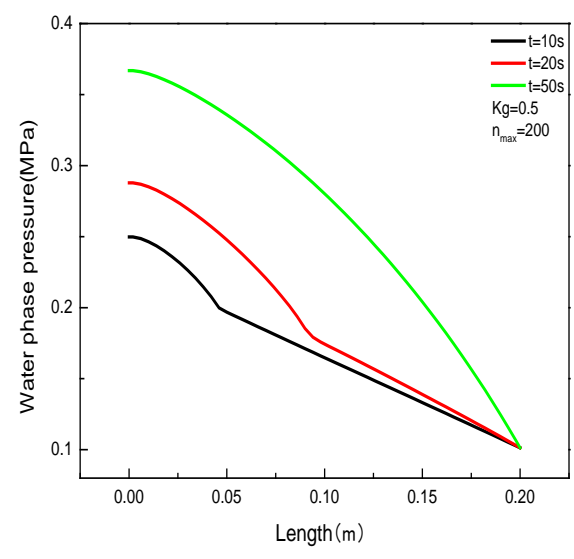

(a)

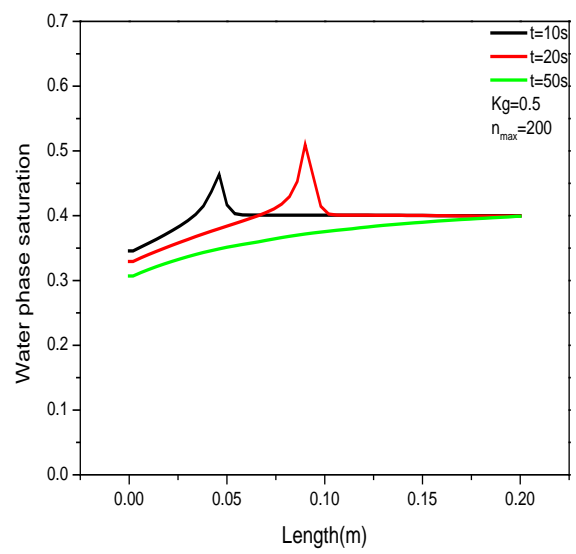

(c)

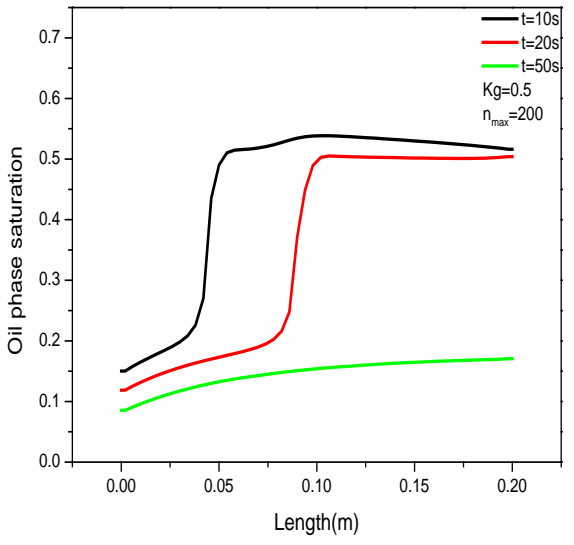

(b)

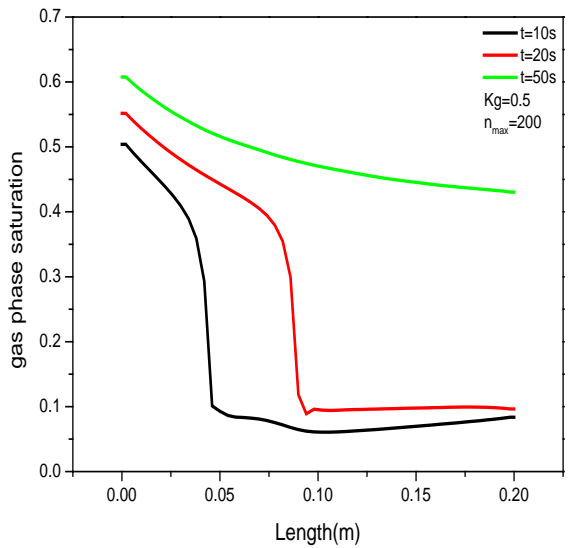

(d)

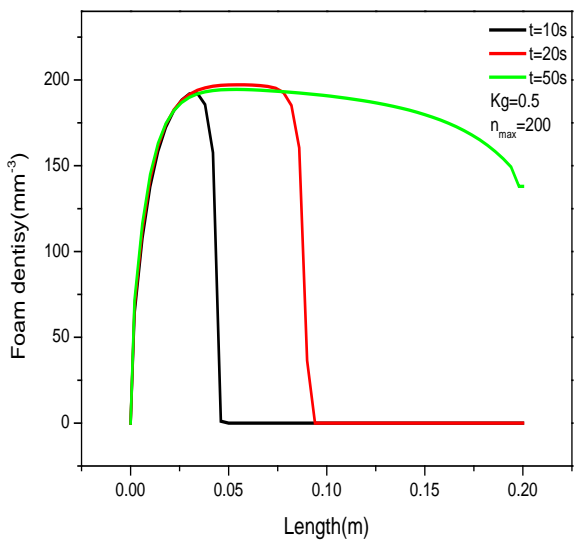

(e)

Fig.2 Different times of displacement (a) water phase pressure (b) oil phase saturation (c) water phase saturation (d) gas phase saturation (e) foam density along the course of change curve $\left(K g=0.5, n_{\max }=200\right)$

\subsection{Foam three phase displacement process under different $\boldsymbol{n}_{\infty}$ values}

Fig.3 (a) to (e) show the water phase pressure, oil/water/gas three phase saturation and foam density curve at displacement time of $\mathrm{t}=20 \mathrm{~s}$ under different $n_{\infty}$ values of 200, 500, 1000 respectively. The numerical simulation 
are carried out at constant $K g=0.5$ to check the effect of $n_{\infty}$ on foam three phase displacement behaviors. Following results can be obtained from the graphs:

(1) The pressure difference of the water phase pressure goes to maximum at $n_{\max }=1000$, which reveals that the parameter $n_{\max }$ mainly affects the apparent viscosity of the foam. The larger the $n_{\max }$, the larger the apparent viscosity of the foam fluid.

(2) With higher pressure drops at larger $n_{\max }$ values, the foam (gas) phase saturation increases, water phase and oil phase saturation decreases, showing an obvious effect of oil recovery enhancement effect.

(3) The bubble density distribution, as shown in Fig.3 (e), doesn't vary too much under different $n_{\max }$ values.

\subsection{Foam three phase displacement process under different $\mathbf{K g}$ values}

Fig. 4 (a)-(e) are the distributive curves of water pressure, oil/water/gas three phase saturation and foam density at displacement time of $\mathrm{t}=20 \mathrm{~s}$ under various $\mathrm{Kg}$ values of $0.1,0.5$ and 1.0 respectively. The numerical simulation are carried out at constant $n_{\max }=200$ to check the effect of $\mathrm{Kg}$ on foam three phase displacement behaviors. Following results can be obtained from the figures:

(1) Different $\mathrm{Kg}$ values have little effect on the water pressure difference in the displacement process under the same $n_{\max }$ values.

(2) The larger the $\mathrm{Kg}$, the greater the foam generation rate, and the foam density can reach the equilibrium value in a relatively short time.

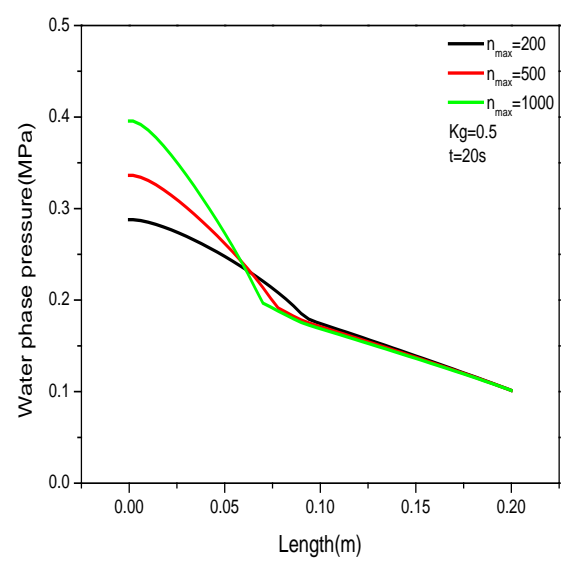

(a)

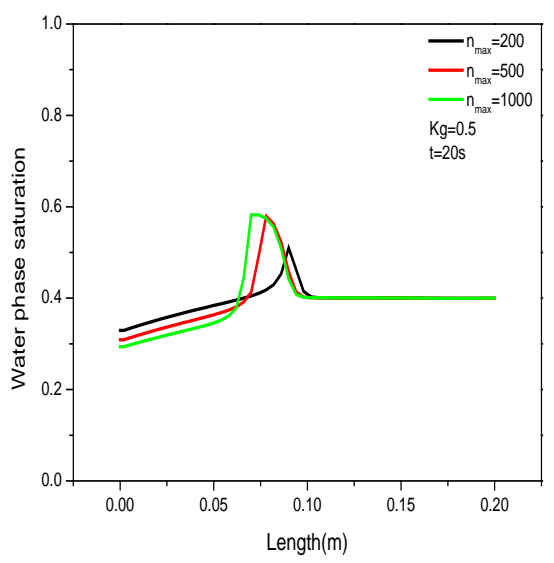

(c)

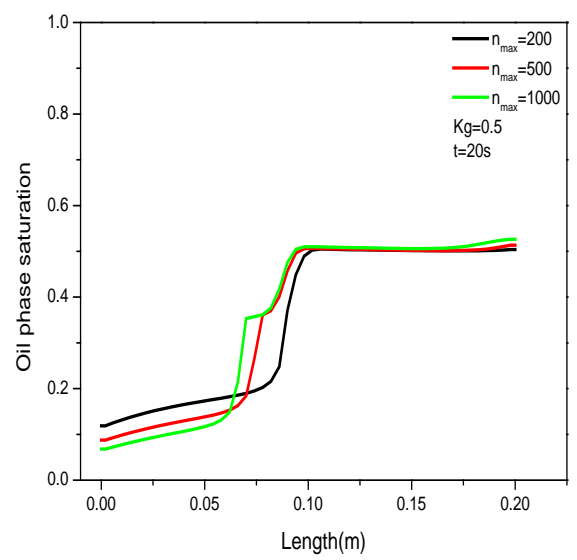

(b)

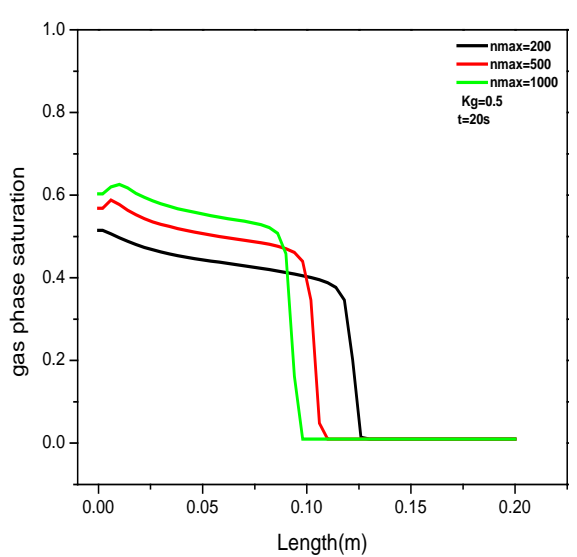

(d) 


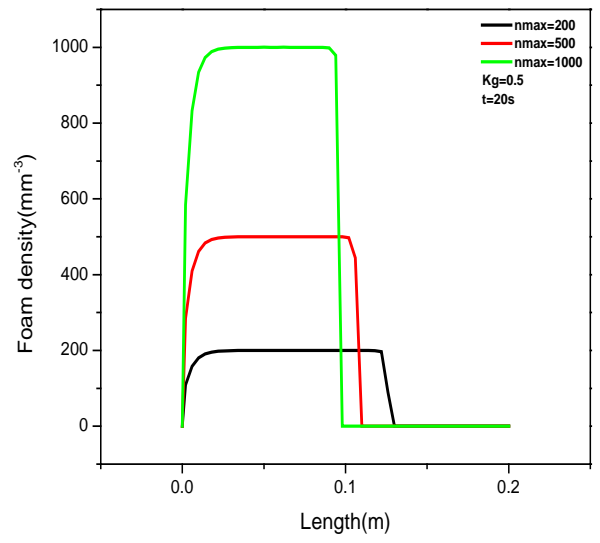

(e)

Fig.3. Foam three phase displacement process under different $n_{\infty}$ values $\left(n_{\max }=200,500,1000\right)$,(a) water pressure (b) oil phase saturation (c) water saturation (d) gas phase saturation (e) foam density curves variation along the course

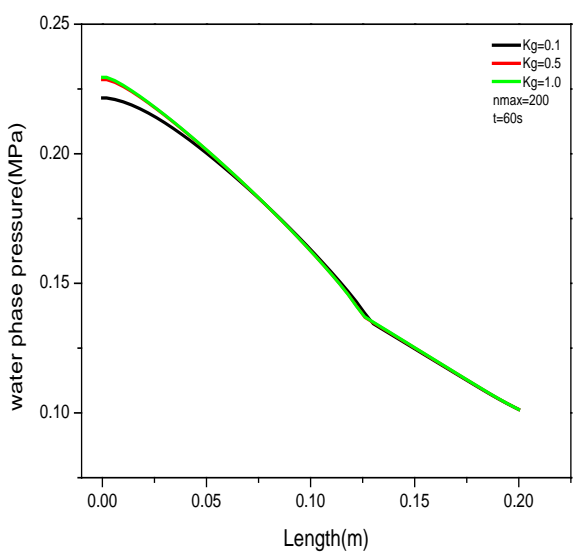

(a)

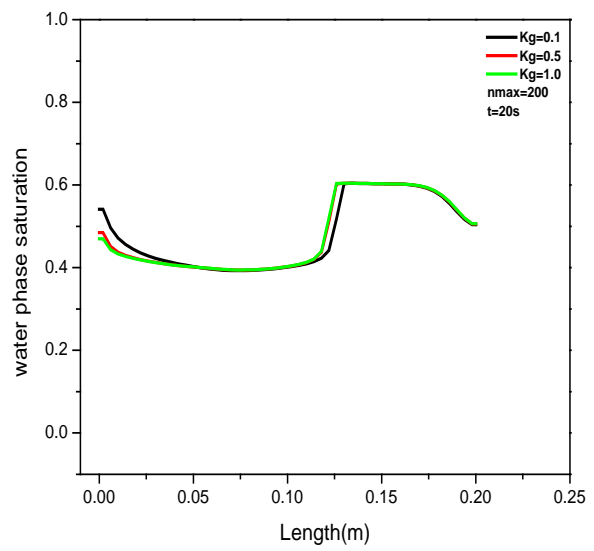

(c)

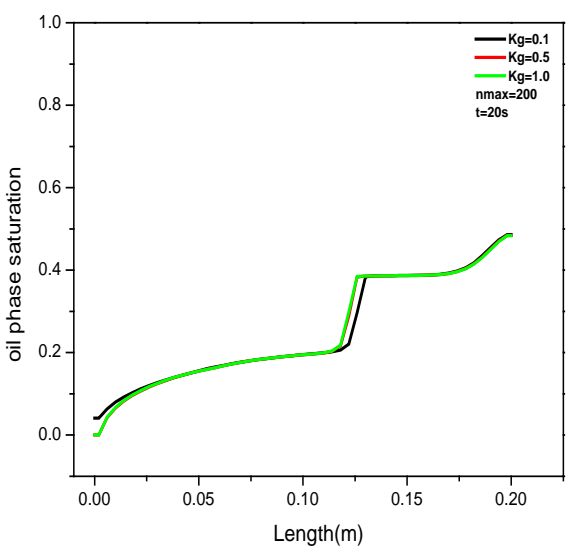

(b)

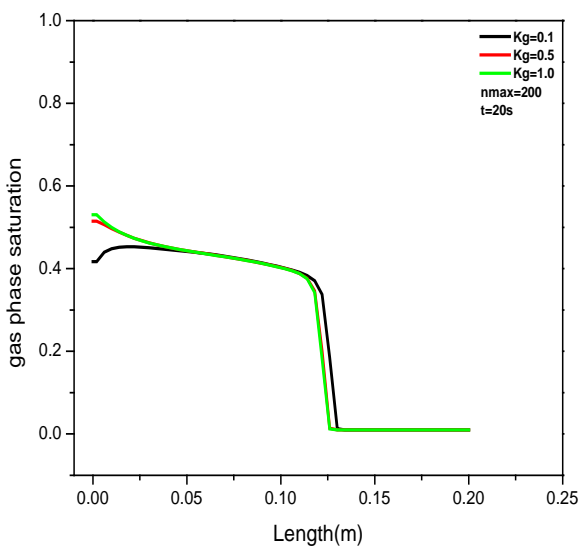

(d) 


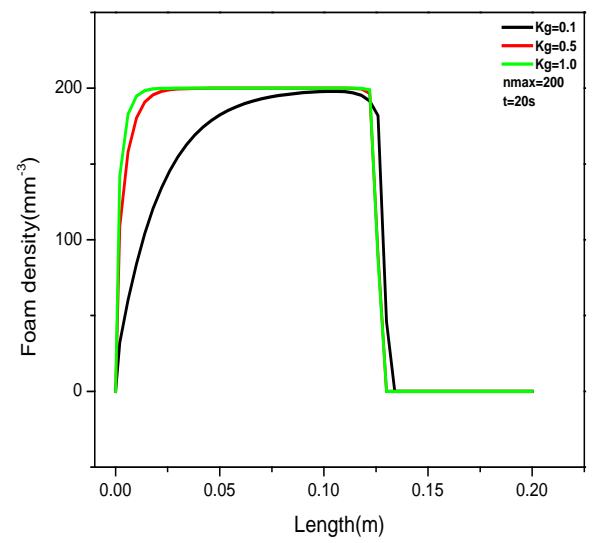

(e)

Fig.4. Foam three phase displacement process under different $K g$ values ( $K g=0.1,0.5,1.0)$, (a) water phase pressure (b) oil phase saturation (c) water phase saturation (d) gas phase saturation (e) foam density curves variation along the course

\section{Conclusions}

In this paper, the stochastic population balance model was employed to simulate the foam three phase displacement behavior in homogeneous porous media. Following conclusions are obtained:

(1)Foam phase could produce extra oil component from oil/gas/water pre-saturated porous media due to its high apparent viscosity;

(2) Elevated maximum foam density $n_{\max }$ leads to higher pressure drop along the sample and therefore results in higher liquid phase recovery rate;

(3)Higher bubble generate rate $\mathrm{Kg}$ values could generate fully developed foam in a shorter distance after gas injection, however shows little effect on total liquid phase displacement efficiency of the foam flooding process.

\section{Acknowledgement}

We gratefully acknowledge the financial support from the National Science Foundation of China (No. 51476081).

\section{References}

[1] Gaspar A.T.F.S., Lima G.A.C. and Suslick S.B. CO2 Capture and Storage in Mature Oil Reservoir: Physical Description, EOR and Economic Valuation of a Case of a Brazilian Mature Field. Paper SPE 94181 presented at SPE Europe/EAGE Annual Conference, Spain, 2005.

[2] Gaspar A.T.F.S., Suslick S.B., Ferreira D.F. and Lima G.A.C. Enhanced Oil Recovery With CO2 Sequestration: A Feasibility Study of A Brazilian Mature Oil Field. Paper SPE 94939 presented at SPE/EPA/DOE Exploration and Production Environmental Conference, Texas, U.S.A., 2005.

[3] Franklin M. and Orr Jr. Storage of Carbon Dioxide in Geologic Formations. Journal of Petroleum Technology, 2004, 56(9): 90.

[4] Moritis G. 2002 Worldwide EOR Survey. Oil and Gas Journal, 2002, 100(15): pp.43-47.

[5] Moritis G. EOR Continues to Unlock Oil Resources. Oil and Gas Journal, 2003, 102(14): pp. 45-49.

[6] Du, D.-X., Zhang, J., Sun, S.-B., Zhang, N., Wang, D.-X., and Li, Y.-G., Mechanistic study on foam lamellae flow characteristics in tubes, Mater. Res. Innovations, vol. 19, no. S5,pp. 526-529, 2015b..

[7] Li Zhaomin, Sun Maosheng, Qi Yuanbing. Journal of Guangxi University (Natural Science Edition), 2006, 31 (3): 212-215.

[8] Li Zhao-Min, LI Song-Yan, SHANG Zhao-Hui, LI Yong. Numerical Simulation of Laminar Jet Flow in Foam Fluids. Drilling Fluid and Completion Fluids, 2007, 24 (1): 58-60, 75.

[9] Du D X, Sun S J, Chen L, Li Y G. Numerical Simulation of Film Foam Flow Characteristics in a Straight Tube, Proceedings of 2009 International Conference on Modeling, Simulation and Optimization, pp.27-30, Beijing, China, 2009.

[10] Rossen W R, Gauglitz P A. Percolation Theory of Creation and Mobilization of Foams in Porous Media.AIChE J. 1990, 36(8): 1176-1188. 
[11] Rossen W R, Shi J, Zeilinger S C. Percolation Modeling of Foam Generation in Porous Media.AIChE J., 1994, 40(6): 1082-1084.

[12] Shi J X. Rossen W R. Simulation of Gravity Override in Foam Processes in Porous Media. SPE Reserv. Eval. and Eng., 1998, 1: 148-154.

[13] Rossen W R, Zeilinger S C, Shi J X, Lim M T. Simplified Mechanistic Simulation of Foam Processes in Porous Media, SPE J., 1999, 4: 279-287.

[14] Cheng L, Kam S I, Delshad M, Rossen W R. Simulation of Dynamic Foam-acid Diversion Processes. SPE J., 2003, 7: 316-324.

[15] Patzek T W. Modeling of Foam Flow in Porous Media by the Population Balance Method. SurfactantBased Mobility Control-ACS Symposium Series 373, 1988, 326-341.

[16] Kovscek A R, Patzet T W, Radke C J. Mechanistic Prediction of Foam Displacement in Multi dimensions: A Population Balance Approach. SPE 27789 in SPE/DOE Ninth symposium on improved oil recovery, Tulsa, USA, 1994.

[17] Kovscek A R, Patzek T W, Radke C J. A Mechanistic Population Balance Model for Transient and Steady State Foam Flow in Boise Sandstone, Chem. Eng. Sci. 1995, 50(23): 3783-3799.

[18] Kovscek A R, Patzek T W, Radke C J. Mechanistic Foam Flow Simulation in Heterogeneous and Multidimensional Porous Media. SPE J., 1997, 2(4): 511-526.

[19] ECLIPSE Simulators. http://www.slb.com/services/software/reseng/blackoil.aspx

[20] CMG Ltd. http://www.cmgl.ca/software/soft-stars

[21] Zitha, P.L.J., D. X. Du. A new stochastic bubble population model for foam flow in porous media[J]. Transp Porous Med, 2010, 83(3), 603-621.

[22] D. X. Du, P. L. J. Zitha, F. J. Vermolen. Numerical analysis of foam motion in porous media using a new stochastic bubble population model[J]. Transp Porous Med,2011,86:461-474.

[23] Du D, Zhang N, Li Y, et al. Parametric studies on foam displacement behavior in a layered heterogeneous porous media based on the stochastic population balance model[J]. Journal of Natural Gas Science \& Engineering, 2017.

[24] Dong-Xing Du, Ali Naderi Beni, Rouhollah Farajzadeh, Pacelli L. J. Zitha. Effect of Water Solubility on Carbon Dioxide Foam Flow in Porous Media: An X-ray Computed Tomography Study. Ind. Eng. Chem. Res.2008,47,6298-6306.

[25] Aziz, K. and Settari, A., Petroleum Reservoir Simulation. Applied Science Publishers Ltd., London UK,(1979)

[26] Diaz-Viera M.A., Lopez-Falcon D.A., Moctezuma-Berthier A., Ortiz-Tapia A., COMSOL Implementation of a Multiphase Fluid Flow Model in Porous Media. Excerpt from the proceedings of the COMSOL Conference 2008 Boston.

[27] Chen, Z., Formulations and numerical methods of the black oil model in porous media, SIAM J. Numer. Anal. 38, 489-514(2000) 